ION CHANNEL STRUCTURE

\section{A new lesson from bacterial channels}

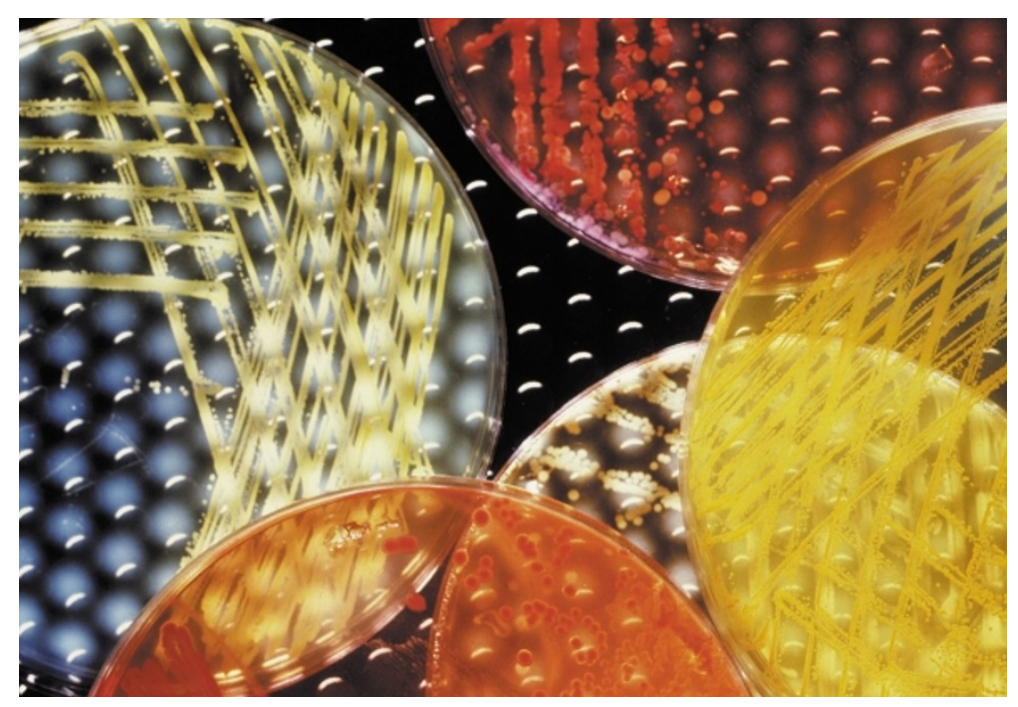

Bacterial ion channels have recently taught us some important lessons on the structure and function of channel proteins from eukaryotic cells, including neurons. First there was KcsA, a bacterial potassium channel that has told us a great deal about the mechanisms of ion selectivity and permeation. Then came GluR0, a prokaryotic glutamate receptor that is permeable to potassium and might represent an evolutionary 'missing link' between ligand- and voltage-gated channels. Enter now $\mathrm{NaChBac}$, a sodium channel from Bacillus halodurans that, surprisingly, is most similar to known voltage-gated calcium channels.

Ren et al. identified $\mathrm{NaChBac}$ while searching for homologues of the sperm protein CatSper, an ion channel that is crucial for male fertility. The amino-acid sequence of $\mathrm{NaChBac}$ is similar to those of voltage-gated calcium channels, particularly in the pore region. But whereas most voltagegated calcium (and sodium) channels have 24 transmembrane helices organized in four homologous domains, $\mathrm{NaChBac}$ has only six, indicating that channel formation requires tetramerization of the protein.

The authors carried out a thorough biophysical characterization of NaChBac channels, and made a series of surprising observations. First, although the pore sequence resembles that of calcium channels, $\mathrm{NaChBac}$ was strongly selective for sodium. Second, although $\mathrm{NaChBac}$ is a sodium channel, it was sensitive to blockers of L-type calcium channels. And third, although the bacterial channel was gated by voltage changes, the kinetics of its activation, inactivation and recovery from inactivation were much slower than those of other voltage-gated sodium channels.

The discovery of $\mathrm{NaChBac}$ tells us that the formation of a sodium channel does not require that the 24 transmembrane domains be present in a single protein molecule; instead, func-

GLIOGENESIS

\title{
Precursors with potential
}

Where do glial cells come from? This is not an easy question to answer, particularly in the context of the complex vertebrate nervous system, but Margot Mayer-Pröschel and her colleagues have made significant progress towards identifying the different glial precursor cells and the lineage relationships between them. A few years ago, they isolated a tripotential glial-restricted precursor (GRP) from the vertebrate spinal cord, which, in vitro at least, can give rise to oligodendrocytes and type 1 and 2 astrocytes. Until recently, the relationship between this cell and other glial precursors was unclear, but the team now report on a new study that might provide some answers.

In vertebrates, it seems that the first step in glial cell differentiation is the generation of multipotent precursor cells, which can give rise to multiple glial lineages but not to neurons. The ability of the GRP cell to generate oligodendrocytes raises fundamental questions regarding its relationship to another oligodendrocyte ancestor, the bipotential oligodendrocyte-type-2 astrocyte progenitor cell (O2A/OPC). Do these distinct precursor cell populations independently generate oligodendrocytes, thereby providing a new example of lineage convergence (the generation of a particular differentiated cell type by more than one developmental lineage), or might there be a direct relationship between them? Gregori et al. examined the lineage relationship between the GRP cell and the $\mathrm{O} 2 \mathrm{~A} / \mathrm{OPC}$, asking the question: do these cells belong to separate but convergent lineages, or is the $\mathrm{O} 2 \mathrm{~A} / \mathrm{OPC}$ derived directly from the GRP cell by a process of lineage restriction?

The authors isolated GRP cells from the embryonic day (E) 13.5 rat spinal cord, and examined their differentiation potential using clonal analysis. They showed that in the presence of platelet-derived growth factor (PDGF) and thyroid hormone (TH), these cells differentiated into cells with characteristics of $\mathrm{O} 2 \mathrm{~A} / \mathrm{OPCs}$; that is, they expressed O4, which is the only antigen known to distinguish O2A/OPCs from GRPs, and they could give rise to oligodendrocytes and type 2 (but not type 1) astrocytes. This indicates that GRPs can act as direct precursors of the O2A/OPCs.

What could be the role of GRPs in vivo? In this study, Gregori et al. explored their potential as oligodendrocyte precursors. Oligodendrocytes are derived predominantly from the ventral spinal cord, but at E13.5, GRPs were present throughout the dorsoventral axis. However, dorsally and ventrally derived GRPs seemed to have different properties. Although they were equally capable of generating O2A/OPCs in the presence of PDGF and TH, the ventrally derived cells were more likely to differentiate along the oligodendrocyte pathway, consistent with earlier observations on the localization of oligodendrocyte production.

So, GRPs are strong candidates for precursors of the oligodendrocyte lineage, with $\mathrm{O} 2 \mathrm{~A} / \mathrm{OPCs}$ acting as intermediates. However, their presence in the dorsal spinal cord, combined with their ability to generate two types of astrocyte in vitro, implies a broader range of functions. Could the GRP cell possibly be the elusive universal glial precursor in the vertebrate nervous system? It is far too early to say, but this work will undoubtedly prompt further studies to show us just what GRP cells are capable of.

Heather Wood

(4) References and links ORIGINAL RESEARCH PAPER Gregori, N. et al. The tripotential glial-restricted precursor (GRP) cell and glial development in the spinal cord: generation of bipotential oligodendrocyte-type-2 astrocyte progenitor cells and dorsal-ventral differences in GRP cell function. J. Neurosci. 22 , 248-256 (2002)

FURTHER READING Zhang, S.-C. Defining glial cells during CNS development. Nature Rev. Neurosci. 2, 840-843 (2001) Lee, J. C. et al. Gliogenesis in the central nervous system. Glia 30, 105-121 (2000) 
tional channels can assemble from simpler building blocks. So, the identification of $\mathrm{NaChBac}$ supports the idea that gene duplications resulted in the appearance of eukaryotic sodium channels. But more importantly, the preference of $\mathrm{NaChBac}$ for sodium, despite its similarity to a calcium channel, should lead us to revise our views on the molecular determinants of ion selectivity. The discovery of this bacterial channel will foster a better understanding of sodium channels, in the same way that KcsA has aided in the study of potassium channels. Juan Carlos López

\section{6) References and links} ORIGINAL RESEARCH PAPER Ren, D. et al. A prokaryotic voltage-gated sodium channel. Science 294, 2372-2375 (2001) FURTHER READING Catterall, W. A. From ionic currents to molecular mechanisms: the structure and function of voltage-gated sodium channels. Neuron 26, 13-25 (2000) WEB SITES

Encyclopedia of Life Sciences http://www.els.net/

ion channels | sodium channels | sodium, calcium and potassium channels

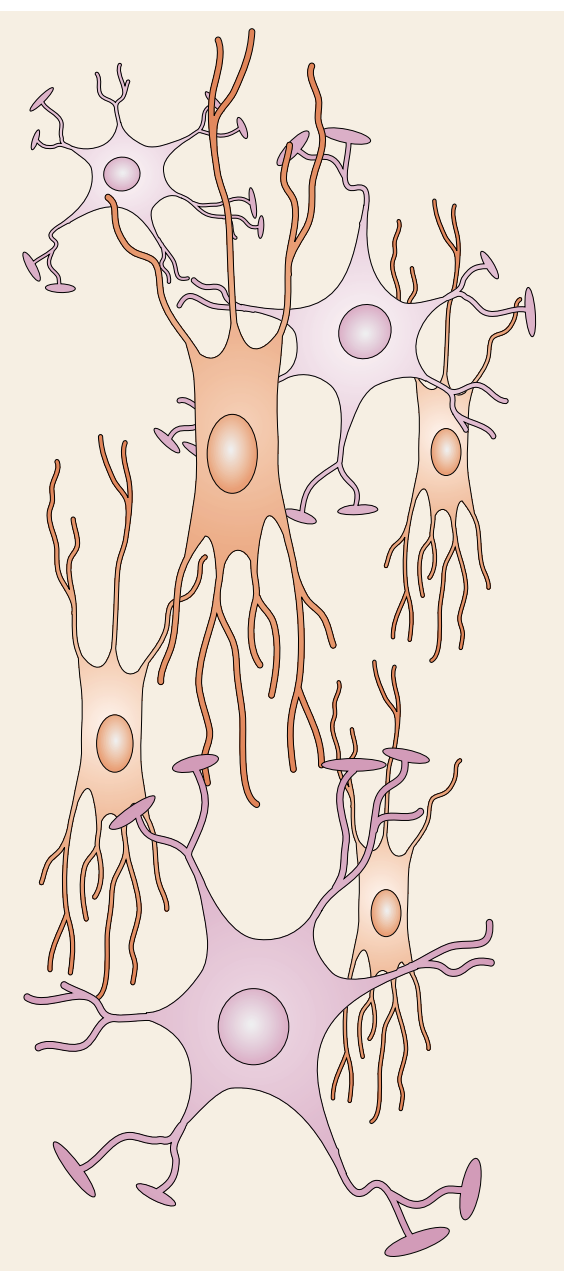

NEUROPROTECTION

\section{New blood in the globin family}

Most of us will have heard of haemoglobin and myoglobin; these porphyrin-containing proteins have a vital role in providing cells with an adequate supply of oxygen. They have been the focus of decades of study, which have taught us an enormous amount about the structure and function of proteins in general, and about the particular roles of globins in vertebrate physiology. By contrast, neuroglobin is a newcomer to the family of vertebrate globins, and clues to its function are only now beginning to emerge. In a report published recently in Proceedings of the National Academy of Sciences, Sun et al. show that this protein might serve to protect neurons against hypoxia.

Owing to the relatively low solubility of oxygen in water, vertebrates rely on oxygen-carrying molecules to maintain aerobic respiration. The heterotetrameric haemoglobins carry oxygen in the blood, whereas myoglobin, a monomer, binds oxygen and facilitates its transport in muscle. Neuroglobin, a monomer with oxygen-binding properties comparable to those of myoglobin, is expressed chiefly in the brain. This fact led Sun and co-workers to examine the possibility that neuroglobin might be involved in neuronal responses to hypoxia and ischaemia.

Using an antibody raised against neuroglobin, Sun et al. showed that when cultures of mouse cortical neurons were deprived of oxygen, expression of the globin increased; the abundance of messenger RNA also increased, in keeping with the transcriptional induction of neuroglobin. Immunocytochemistry revealed that this protein was present in the cytoplasm of cells that expressed the neuronal nuclear antigen $\mathrm{NeuN}$ and were therefore likely to be neurons. And in immunostained sections of the cerebral cortex of rodents that had been subjected to focal cerebral ischaemia, Sun et al. showed that neuroglobin expression in the cytoplasm of cortical neurons was increased in the ischaemic compared with the non-ischaemic hemisphere.

But could neuroglobin protect neurons against hypoxia? Sun et al. transfected cultured neurons with an antisense oligodeoxynucleotide directed against the initial coding region of neuroglobin mRNA. As expected, the antisensetransfected cells, which expressed lower levels of neuroglobin than sense-transfected or untreated cells, were more susceptible to hypoxia.

Conversely, overexpression of neuroglobin in an immortalized hippocampal neuronal cell line

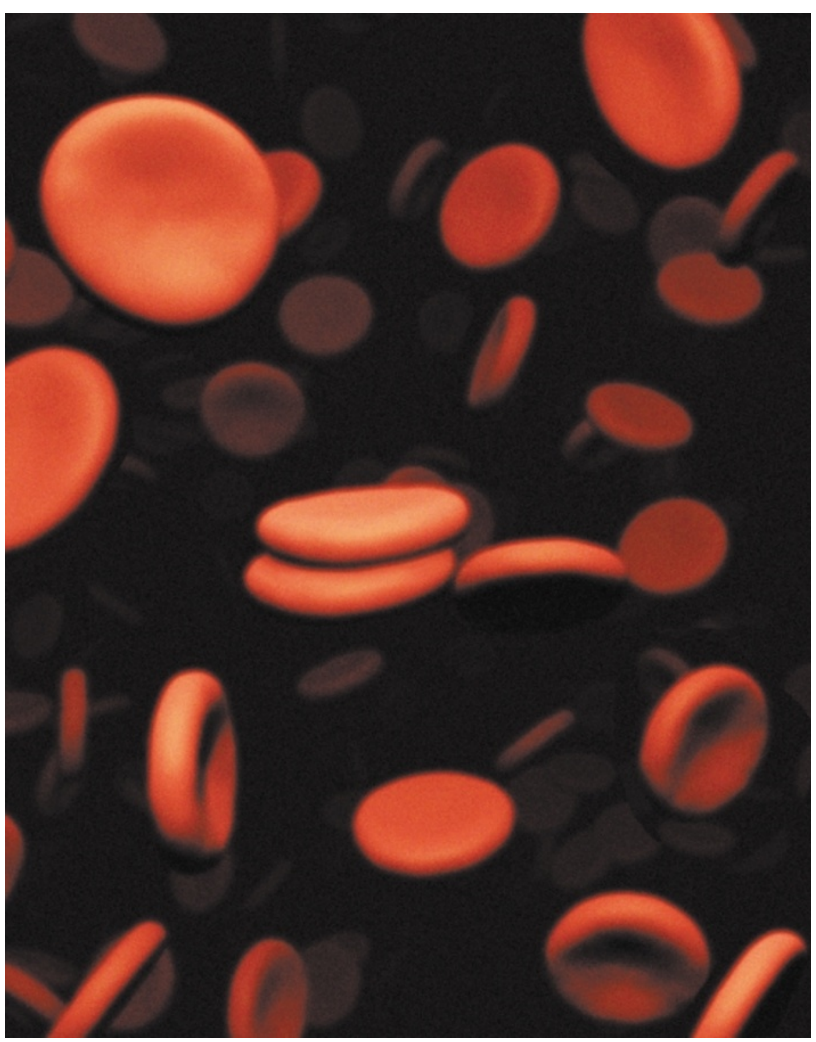

increased the viability of cells that were exposed to hypoxia.

So, one role of neuroglobin seems to be to promote neuronal survival after hypoxicischaemic insults. It remains to be seen how neuroglobin exerts its neuroprotective effect. Although globins are perhaps best known for their roles as oxygen carriers, these proteins have assumed a range of functions in evolution, including the scavenging and sensing of gases such as nitric oxide and carbon dioxide. So, for example, neuroglobin might act by facilitating the transport of oxygen to mitochondria, by scavenging the mediators of hypoxic-ischaemic injury, or by sensing hypoxia and activating protective mechanisms.

Neuroglobin has a long way to go to match the prominence of haemoglobin and myoglobin in the biosciences; nevertheless, Sun et al. have made a significant advance in defining a role for this protein. An important next step will be to determine whether neuroglobin has a neuroprotective effect in vivo; for example, by showing that cortical neurons are less sensitive to focal cerebral ischaemia in animals that overexpress this protein.

Rebecca Craven

(9) References and links

ORIGINAL RESEARCH PAPER Sun, Y. et al. Neuroglobin is

up-regulated by and protects neurons from hypoxic-ischemic injury.

Proc. Natl Acad. Sci. USA 98, 15306-15311 (2001)

FURTHER READING Burmester, T. et al. A vertebrate globin

expressed in the brain. Nature 407, 520-523 (2000)

WEB SITES

David Greenberg's lab: http://www.buckinstitute.org/ 